\title{
Étude d'une géométrie innovante pour le refroidissement de plaques de piles à combustible PEMFC
}

\author{
Nadia Caney ${ }^{1, a}$, Alain Maréchal ${ }^{2}$ et Philippe Marty ${ }^{1}$ \\ 1 LEGI-GRETh, 17 rue des martyrs, 38054 Grenoble Cedex 9, France \\ 2 CEA GRETh, 17 rue des martyrs, 38054 Grenoble Cedex 9, France
}

Reçu le 15 mars 2007, accepté le 7 juin 2007

\begin{abstract}
Résumé - Le refroidissement d'une pile à combustible (PEM), utilisée comme source d'énergie électrique dans le domaine de la traction automobile, fait apparaître un certain nombre de contraintes. Une des contraintes fortes se trouve être le refroidissement du cœur de pile, notamment du fait que le niveau de température est faible (environ $80^{\circ} \mathrm{C}$ ), mais aussi que la puissance thermique à évacuer est élevée. Cette étude s'intéresse à un refroidissement de la pile par l'évaporation interne d'un fluide ou par circulation d'un fluide monophasique. Le dispositif expérimental permet de vaporiser le fluide, le HFE7100, le long d'une plaque dont on souhaite mesurer les performances. Une technologie innovante de plaque présentant des bossages ou picots inversés est proposée. Ainsi, cette géométrie est très performante pour du refroidissement monophasique mais l'optimisation reste à faire en régime diphasique.
\end{abstract}

Mots clés : Refroidissement monophasique / diphasique / pile à combustible / échange thermique / pertes de pression

\begin{abstract}
Study of a new geometry for fuel cells cooling. Use of an embarked fuel cell, especially in a car, requires improving the cooling system efficiency. In such an application, one of the constraints is the low temperature $\left(80{ }^{\circ} \mathrm{C}\right)$ and the important heat power to dissipate. A solution is to extract the latent heat of a boiling fluid. Another solution is to use a single phase flow fluid for cooling the system. For these reasons, heat transfer coefficient and frictional pressure drop are experimentally investigated. Single phase flow and flow boiling configurations of HFE7100 are tested in a vertical mini-channels device. An innovative technology is proposed. This geometry well performs for single phase flow and has to be improved for two-phase flow.
\end{abstract}

Key words: Single phase flow cooling / two-phase flow / fuel cell / heat transfer / pressure drop

\section{Introduction}

En vue de la présentation d'une géométrie nouvelle optimisée pour le refroidissement de pile à combustible, nous proposons tout d'abord une étude sur une géométrie qui servira de référence. Cette géométrie est constituée d'une plaque à canaux droits sur laquelle les calculs d'échanges thermiques en local sont plus aisés. Elle permet de tester sur une géométrie simple, les critères d'homogénéité et de température maximale atteinte. Ensuite, les essais expérimentaux seront comparés à ceux obtenus pour la nouvelle technologie proposée.

\footnotetext{
a Auteur pour correspondance : nadia.caney@cea.fr
}

\subsection{Présentation des outils expérimentaux}

Les éléments suivants composent le circuit principal du dispositif expérimental (cf. Fig. 1) :

- la section d'essais,

- un débitmètre massique Coriolis de gamme 0$160 \mathrm{~kg} \cdot \mathrm{h}^{-1}$ (précision de 0,2\%) et une pompe de type volumétrique à vitesse variable de 16 à $1566 \mathrm{ml} . \mathrm{min}^{-1}$,

- deux résistances électriques montées en parallèle enroulées autour d'un tube inox qui préchauffent le fluide à la température désirée,

- un réservoir condenseur d'un volume de $0,5 \mathrm{l}$ et un échangeur à contre-courant chargé de sous-refroidir le liquide en entrée de la pompe.

La première section d'essais (notée plaque 1, cf. Fig. 2) est composée de 40 canaux parallèles d'épaisseur $0,5 \mathrm{~mm}$ 


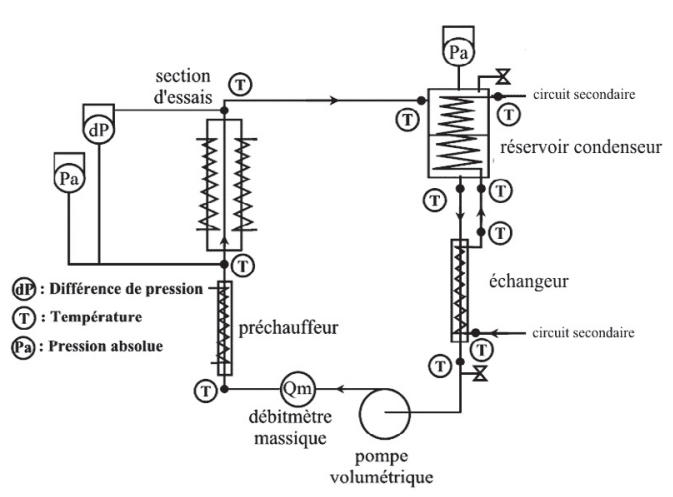

Fig. 1. Schéma de principe simplifié du banc d'essais.

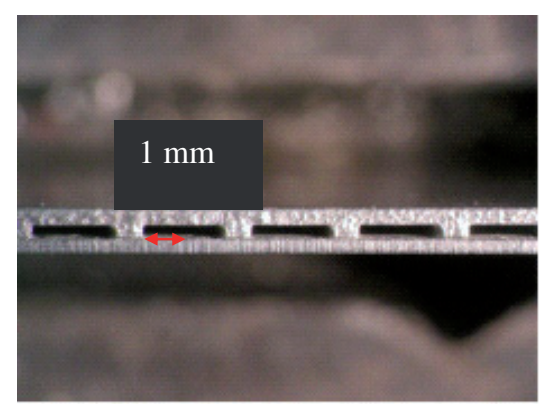

Fig. 2. Plaque 1 à canaux droits.

d'une largeur de 2,6 $\mathrm{mm}$ espacés de $1 \mathrm{~mm}$ alimentés par le fluide en ébullition. Le chauffage du fluide est assuré par un film chauffant. Le titre à l'entrée des canaux est déterminé à l'aide d'un bilan thermique sur le préchauffeur : les valeurs locales de titre et de température le long du canal sont ainsi déduites en supposant le flux homogène sur toute la plaque; cette hypothèse a été étayée par des mesures de caméra infrarouge. La puissance thermique dégagée par ce dernier est déduite de la mesure de l'intensité et de la tension aux bornes du film résistif. Cette puissance varie entre 0 et $220 \mathrm{~W}$. Deux sondes PT100 de 0,5 mm de diamètre (précision de $\pm 0,15^{\circ} \mathrm{C}$ ) mesurent les températures d'entrée et de sortie du fluide. Les températures de paroi sont mesurées à l'aide de thermocouples de type $\mathrm{K}$ de diamètre $0,25 \mathrm{~mm}$, insérés dans des rainures de 0,3 $\mathrm{mm}$ de section. Ainsi, la section d'essais comporte 11 thermocouples disposés de part et d'autre de la plaque permettant de suivre l'évolution de la température au voisinage de 3 canaux.

La plaque à picots (plaque 2) est constituée de 2 tôles, formées par un procédé d'emboutissage ou d'estampage; la face d'une tôle est ainsi le «négatif » de son autre face. Les emboutissages de forme cylindrique forment des picots et sont disposés en quinconce sur chacune des 2 tôles. L'assemblage de ces deux tôles l'une sur l'autre, avec un décalage donné, définit un réseau de canaux permettant le passage du fluide de refroidissement entre les plaques. Malheureusement, la technique d'emboutissage n'est pas du tout adaptée à l'obtention de pièce unitaire ou de très petite série, compte tenu du coût d'investissement de l'outillage. Il a donc été envisagé de construire une plaque

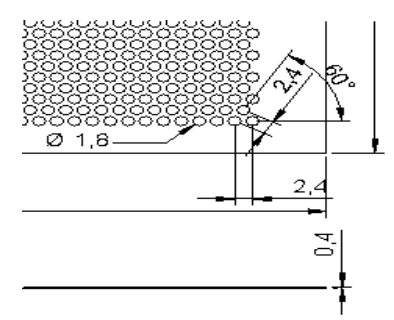

Fig. 3. Perçage d'une tôle.

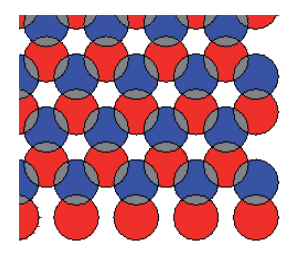

Fig. 4. Schéma de superposition de 2 tôles.

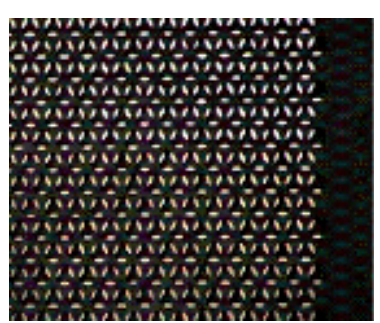

Fig. 5. Photo de la superposition de 2 tôles.

similaire par un usinage classique. Elle est constituée par la superposition de quatre tôles (cf. Figs. 3-5) :

- deux tôles d'épaisseur 0,4 $\mathrm{mm}$ sont percées de 10117 trous de diamètre $1,8 \mathrm{~mm}$ selon un motif triangulaire de pas $2,4 \mathrm{~mm}$; ces deux tôles sont assemblées avec un décalage de 0,52 $\mathrm{mm}$;

- cet ensemble est ensuite recouvert latéralement de deux tôles de faible épaisseur $(0,1 \mathrm{~mm})$, déterminant ainsi un réseau interne de canaux.

Les obstacles ainsi créés sont répartis de façon homogène sur toute la surface de la plaque, et suggèrent que le cheminement du fluide est globalement mono directionnel.

\section{Résultats des essais en écoulement monophasique}

Pour les deux plaques, les pertes de pression sont mesurées entre l'amont du collecteur d'entrée et l'aval du collecteur de sortie. Les pertes de pression sont donc globales et tiennent compte à la fois de la perte de pression singulière des collecteurs/distributeurs et de la perte de pression régulière dans la zone thermiquement active.

La figure 6 fait clairement apparaître le point suivant : la plaque à picots génère plus de pertes de pression que la plaque 1 . On peut penser que les pertes de pression de cette nouvelle géométrie sont probablement occasionnées 


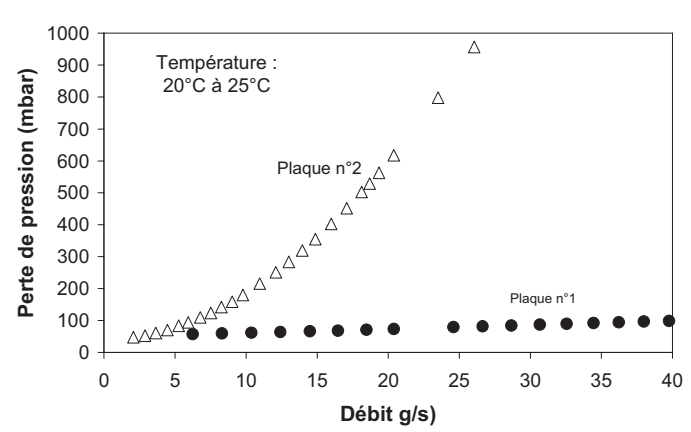

Fig. 6. Pertes de pressions mesurées aux bornes des deux géométries.

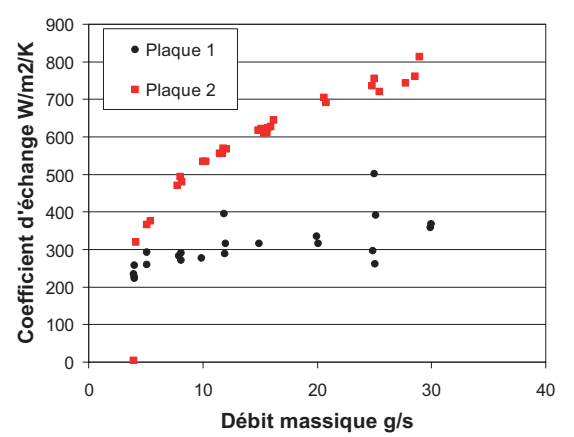

Fig. 7. Coefficient de transfert de chaleur global des deux plaques.

par les changements de direction continuels du fluide entre les picots, plus que par la composante singulière en entrée et sortie.

Concernant le coefficient de transfert de chaleur global sur la partie thermiquement active de chaque plaque (Fig. 7), on observe la même hiérarchie que pour les pertes de pression. La plaque 1 est la moins gourmande en terme de pertes de pression et la moins efficace en échange de chaleur. Pour un débit entre 10 g.s ${ }^{-1}$ et 15 g.s ${ }^{-1}$, les niveaux d'échange sont respectivement de $350 \mathrm{~W} \cdot \mathrm{m}^{-2} . \mathrm{K}^{-1}$ pour la première plaque et de $600 \mathrm{~W} \cdot \mathrm{m}^{-2} \cdot \mathrm{K}^{-1}$ pour la seconde. Il est assez normal de trouver la plaque 1 la plus mal placée d'un point de vue thermique. En effet, le fluide s'écoule dans des canaux droits sans aucun brassage. Pour la plaque 2, les changements continuels de direction permettent d'améliorer le transfert de chaleur par brassage du fluide.

\section{Résultats des essais en diphasique}

\subsection{Coefficient d'échange global}

La plaque 2 génère nettement plus de pertes de pression (cf. Fig. 8). Le coefficient de transfert de chaleur global présente cette fois une hiérarchie inversée par rapport à celle des pertes de pression (cf. Fig. 9). La plaque qui donne le moins de pertes de pression donne le meilleur transfert de chaleur et, à l'opposé, la plaque qui donne le plus de pertes de pression donne l'échange le moins efficace. Pour chacune des 2 plaques, le niveau d'échange

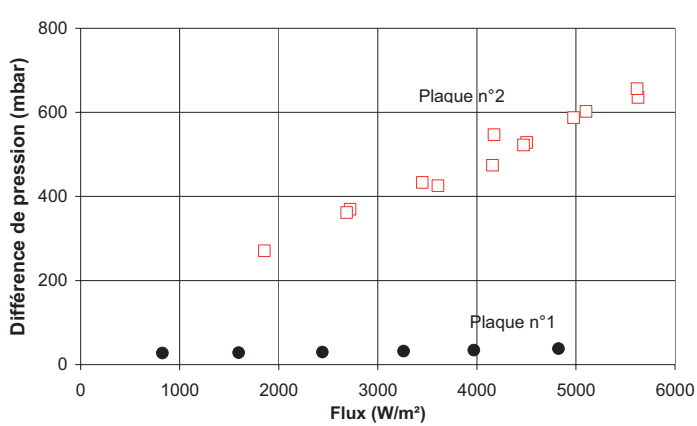

Fig. 8. Pertes de pressions en fonction du flux de chaleur pour un débit constant de 4 g.s ${ }^{-1}$.

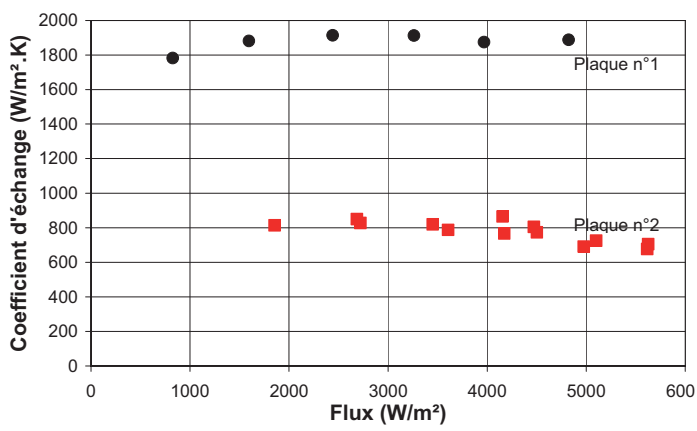

Fig. 9. Coefficient de transfert de chaleur global.

thermique est indépendant du flux de chaleur appliqué sur la plaque.

\section{2 Écarts de température}

Pour un bon fonctionnement de la pile, un critère d'homogénéité a été fixé à un écart de $2{ }^{\circ} \mathrm{C}$ maximum (cf. Fig. 10).

- La plaque 1 est celle qui présente un écart minimum de température. Par exemple pour un flux de chaleur de $3000 \mathrm{~W} . \mathrm{m}^{-2}$, l'écart maximum est de l'ordre de $0,5{ }^{\circ} \mathrm{C}$.

- La plaque 1 est remarquable vis-à-vis du critère d'homogénéité de température de paroi puisqu'elle donne un écart de température inférieur à $1^{\circ} \mathrm{C}$, quelle que soit la valeur du flux de chaleur.

La perte de pression dans la partie active de la plaque 2 provoque une évolution importante de la température du fluide entre l'entrée et la sortie. La température du fluide suit la loi de température de saturation en fonction de la pression de saturation. Par conséquent la température de paroi va elle aussi présenter une variation importante.

Concernant la température maximum de paroi (Fig. 11), les essais sont réalisés en maintenant la pression de sortie constante au voisinage de la pression atmosphérique. Compte tenu de la perte de pression globale pour chaque plaque c'est la pression à l'entrée de la plaque 2 qui est la plus élevée. En fonction des remarques précédentes, le niveau de température de paroi 


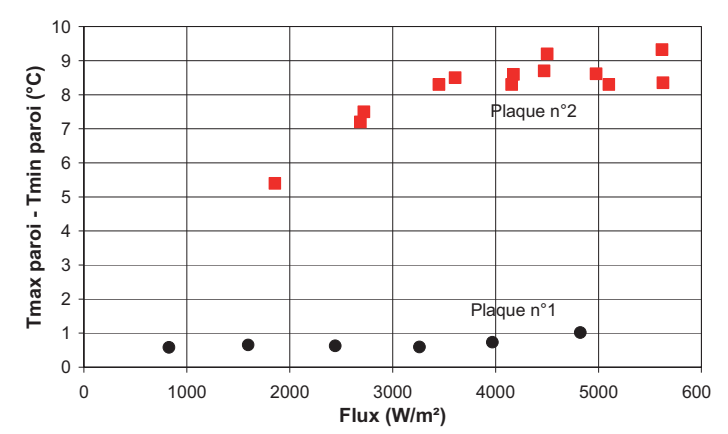

Fig. 10. Écart maximum de température de paroi en fonction du flux de chaleur et pour un débit constant de 4 g.s ${ }^{-1}$.

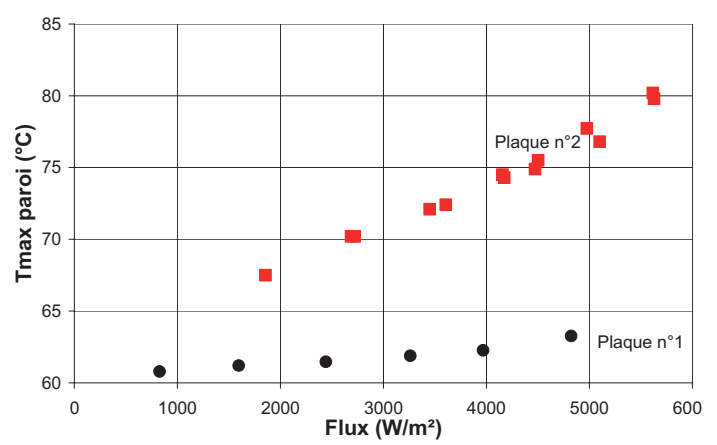

Fig. 11. Température de paroi maximum atteinte en fonction du flux de chaleur.

de plaque est donné par le niveau de température de fluide à l'entrée (loi de température de saturation en fonction de la pression de saturation). En conséquence la plaque 2 présente une température maximum de surface supérieure à la plaque 1 .

\subsection{Coefficient d'échange local}

Les mesures de température le long de la plaque permettent une analyse locale du transfert de chaleur. Le coefficient de transfert local est présenté sur la figure 12 pour la plaque 2. On observe que, quel que soit le débit massique ou le flux thermique imposé, passée l'entrée de la plaque, la valeur du coefficient d'échange est très faible par rapport à ce qu'on pourrait attendre d'un transfert en évaporation. La chute du coefficient d'échange intervient même à des faibles titres, compris entre 0,1 et 0,2 . Ceci est représentatif du phénomène d'assèchement de la paroi, celle-ci n'étant plus en contact avec un film liquide : ceci a pour effet d'augmenter fortement la température de paroi. La figure 13 extraite de [1] montre que pour la plaque 1, dans les mêmes conditions expérimentales, le coefficient d'échange local se situe entre 2000 et $4000 \mathrm{~W} \cdot \mathrm{m}^{-2} \cdot \mathrm{K}^{-1}$. Une chute à des valeurs inférieures à $1000 \mathrm{~W} \cdot \mathrm{m}^{-2} \cdot \mathrm{K}^{-1}$ est observée.

Il semble probable que ce n'est pas l'augmentation du titre massique de vapeur qui gouverne la chute du coefficient d'échange local, mais plutôt l'organisation interne de l'écoulement. Il semble que les multiples changements de direction du fluide, à l'intérieur des picots de la plaque 2 ,

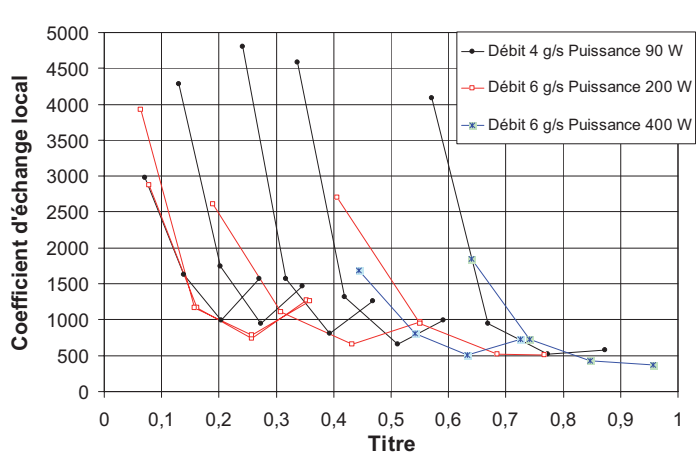

Fig. 12. Plaque 2, analyse locale du coefficient d'échange.

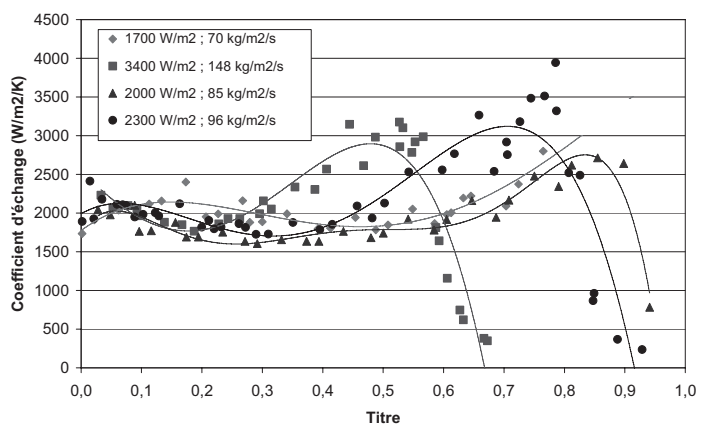

Fig. 13. Plaque 1, analyse locale du coefficient d'échange.

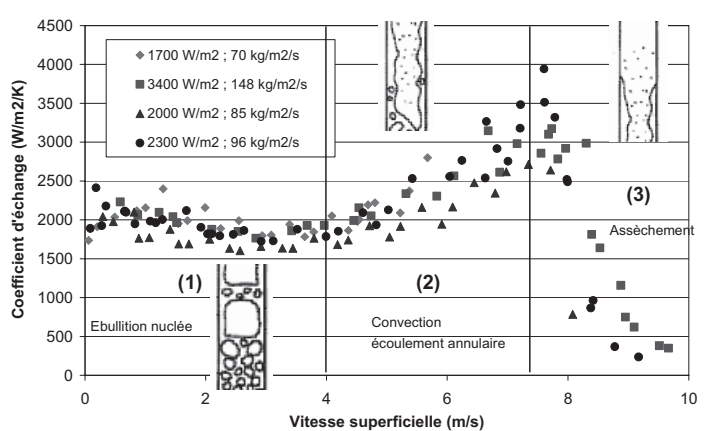

Fig. 14. Analyse locale du coefficient d'échange en fonction de la vitesse superficielle.

ne sont pas du tout favorables au développement d'un film liquide en paroi, ce qui explique la dégradation du coefficient de transfert local.

Une analyse locale plus poussée [2] nous a permis d'analyser le comportement de la plaque 1. En effet, si le coefficient d'échange est tracé en fonction de la vitesse moyenne de mélange ou vitesse superficielle plutôt que le titre, les points expérimentaux se regroupent et il est possible de mieux distinguer les configurations d'écoulement (cf. Fig. 14).

De plus, l'assèchement correspondant à la chute du coefficient d'échange se produit maintenant pour une vitesse $J$ comprise entre 7 et $8 \mathrm{~m} . \mathrm{s}^{-1}$. Il est ainsi possible de distinguer trois zones dans l'évolution du coefficient d'échange :

- Pour des faibles titres, la valeur du coefficient d'échange est stable ce qui correspond à l'évolution classique de l'échange thermique en ébullition nucléée. 
- À partir d'une certaine vitesse (environ $5 \mathrm{~m} . \mathrm{s}^{-1}$ ), le coefficient d'échange augmente. Cela peut s'expliquer comme le début de la transition vers l'écoulement annulaire. En effet, les bulles ont atteint une certaine taille et commencent à coalescer entre elles pour former des bouchons de plus en plus gros. Il y a alors apparition d'un film liquide en paroi dont l'épaisseur va diminuer progressivement, c'est le régime d'ébullition convective.

- Enfin, à partir d'une certaine valeur de la vitesse, le film liquide ne peut plus se maintenir en paroi. La vitesse de la vapeur vient alors arracher le liquide ce qui a pour conséquence de faire chuter brutalement le coefficient d'échange, cela pour $J=7$ à $8 \mathrm{~m} . \mathrm{s}^{-1}$.

\section{Conclusion et perspectives}

La géométrie de la plaque à canaux droits s'avère la plus performante vis-à-vis du refroidissement diphasique et des pertes de pression. Par conséquent elle est la plaque de référence car elle satisfait les critères d'homogénéité et de température maximale atteinte. Malheureusement cette géométrie semble difficilement applicable à une plaque bipolaire qui doit être de conception emboutie pour être techniquement réalisable. Enfin, les pertes de pression obtenues avec la géométrie à picots inversés sont supérieures à celles de la plaque 1. De plus, l'homogénéité de température de surface n'est pas satisfaisante. La température maximale atteinte reste compatible avec les températures de fonctionnement d'une membrane, et les pertes de pression sont uniformément distribuées à l'intérieur de la plaque.

Du fait du mode de fabrication de cette plaque, les picots ont une forme cylindrique alors qu'ils auraient été de forme tronc conique si la plaque avait été emboutie. Malgré tout, cette première géométrie semble adaptée pour du refroidissement monophasique.

En régime de refroidissement diphasique, l'optimisation reste à faire et doit porter sur les points suivants :

- augmenter le diamètre des trous,

- réaliser une plaque avec des picots de forme conique,
- réfléchir aux alimentations des gaz et du fluide de refroidissement,

- optimiser la forme du picot (par exemple, allongé dans le sens de l'écoulement principal),

- adapter la forme et la taille des picots par zone dans la plaque.

Le problème de la distribution diphasique dans une PAC qui comporte environ une centaine de plaques en parallèle doit être étudié attentivement afin d'assurer un débit identique dans chaque plaque.

Concernant l'aspect thermique, et en particulier les résultats de la plaque 1 , le paramètre important pour le dimensionnement se révèle être la vitesse superficielle. Trois zones d'échange thermique ont pu être identifiées en fonction de ce paramètre : la première pour des vitesses inférieures à $5 \mathrm{~m} . \mathrm{s}^{-1}$, où le coefficient d'échange varie peu; une seconde pour des vitesses comprises entre 5 et $8 \mathrm{~m} . \mathrm{s}^{-1}$ où l'écoulement devient annulaire et le coefficient d'échange augmente avec la vitesse et qui correspond à une zone d'ébullition convective; enfin une troisième zone de démarrage d'assèchement pour des vitesses supérieures à $8 \mathrm{~m} . \mathrm{s}^{-1}$ où le coefficient d'échange chute brutalement. Les résultats les plus récents dans les mini et micro-canaux sont en accord avec cette évolution du coefficient d'échange en trois zones distinctes. De plus, certaines études en visualisation ont permis d'identifier clairement les transitions entre les deux zones d'ébullition nucléées et convectives. Concernant l'apparition de la zone d'assèchement, elle est plus difficile à caractériser mais certains auteurs ont réussi à montrer qu'elle dépend fortement de la géométrie. Aucune étude n'a mis encore en évidence l'influence à la fois de la structure de la paroi, de la géométrie et de la nature du fluide ce qui laisse envisager d'importantes perspectives.

\section{Références}

[1] F. Madrid, N. Caney, P. Marty, Flow boiling study in minichannels, Heat transfer engineering, 2007, à paraître

[2] N. Caney, P. Marty, J. Bigot, Experimental flow boiling in mini-channels, International Heat Transfer Conference, Sydney, Australia, 13-18 August 2006 